\title{
Move backward, forward signals
}

\author{
Peter Nick ${ }^{1}$
}

Received: 6 August 2019 / Accepted: 6 August 2019 / Published online: 10 August 2019

(C) Springer-Verlag GmbH Austria, part of Springer Nature 2019

Microtubules exhibit a distinct directionality, which is read out as directional movement by the molecular motors kinesin and dynein motors (Hyman and Mitchison 1991). While kinesins move towards the growing end of the microtubules, dynein motors move in the opposite direction. Dynein motors are also coupled to actin via the dynactin complex (Allan 1994), which allows establishing a tensegral network, which is able to integrate mechanic forces. Thus, this system of tracks and motors allows generating direction and, therefore, plays an important role in development. For instance, kinesin motors help to define the posterior pole of the fruit fly embryo by transporting mRNA coding for the morphogenetic determinants oskar and nanos (Clark et al. 1994), or the dynein motor inversus viscerum defines the left-right asymmetry of mice (Supp et al. 1997). While kinesin motors follow the growing tip and, thus, project the process of microtubule growth into space, dynein motors move towards the site, where the microtubule was nucleated originally, and thus move back into history, which allows integrating time, while the tensegrity through cross-linking to actin filaments allows integrating space. Two contributions to the current issue deal with the role of minus-end-directed motors in biological signalling.

The work by Fokin et al. (2019) in the current issue is focussed on the centrosome, the major microtubuleorganising centre of animal cells that during interphase organises a radial microtubule aster. The authors pursue the working model that this aster is relevant for the primary cilium, a rudimentary organelle remaining from the flagellate ancestral state of all animal cells, which has undergone a functional shift from mobility towards signalling. The link between centrosome and primary cilium is the protein Pericentriolar Matrix 1 (PCM-1), which participates in the organisation of the primary cilium, but also is part of the pericentriolar satellites. The

Peter Nick

peter.nick@kit.edu

1 Botanical Institute, Karlsruher Institut für Technologie, Karlsruhe, Germany correct localisation of PCM-1 to the periphery of the centriole is brought about by dynein, acting in the context of the dynactin complex. The authors show that this movement requires the activity of a specific kinase that phosphorylates the p150Glued subunit of dynactin and, thus, releases the cargo (PCM-1) for minus-end-directed transport. Using specific inhibitors and dominant-negative mutants of p150Glued, they demonstrate that disruption of this kinase activity not only causes diffuse spread of PCM-1 over the entire cytoplasm (which is expected as direct consequence), but also interferes with the organisation of the primary cilia (which represents a major implication of their model). Although in mammals cilia or flagella are not required any longer for mobility (with exclusion of the sperm, which recapitulates the ancestral situation), ciliopathies have severe consequences, because the primary cilium conveys important functions in the organisation of signalling. Thus, the centrosome, in addition to recruiting its own components through a minus-end-directed microtubule motor (the authors use the term egoistic function), uses the same machinery to organise other organelles, such as the primary cilium (which would represent an altruistic function of the centrosome). The partitioning between egoistic and altruistic functions must be regulated, for instance, by kinase cascades as those dissected by the authors.

Also, the work by Spiegelman et al. (2019) in the current issue deals with the role of minus-end-directed microtubule motors for signalling. However, the model (higher plants) and the context (intercellular signalling) differ. Moreover, there are no dyneins involved - these have been lost during the evolution of terrestrial plants. The last species still harbouring a dynein is the maidenhair tree Ginkgo biloba, which needs to run the cilia of a spermatozoid to bridge the last $50 \mu \mathrm{m}$ from the bursting pollen tube to the egg cell. While dyneins progressively have left the scene, a new group of class-XIV kinesins emerged and rapidly diversified. These $\mathrm{KCH}$ kinesins are not following the canonical behaviour, but move to the minus-end of microtubules, and they harbour a so called calponin-homologue $(\mathrm{CH})$ domain which cross-connects these kinesins with actin filaments. In other words, the $\mathrm{KCH}$ 
kinesins might be the plant functional analogues of the dynein-dynactin machinery found in animal cells. The authors focus on one specific $\mathrm{KCH}$, Kinesin $\mathrm{G}$, which was identified as interactor for a regulator for SHORTROOT. The transcription factor SHORTROOT is formed in the central region of the root meristem and then transported centrifugally into the neighbouring cell layer, where it drives the differentiation of endodermal tissue. By constitutive overexpression, a root with ectopic layers of endodermis can be generated (Nakajima et al. 2001). During the attempt to understand the mechanism behind this intercellular transport, the authors had worked on an endosome-associated protein termed SHR-INTERACTINGEMBRYONIC-LETHAL (SIEL), and later identified Kinesin $G$ as interactor of SIEL that was essential for correct intercellular transport of SHORTROOT (Spiegelman et al. 2018). In the current work, they address the role of the endoplasmic reticulum for this functional context. They demonstrate that both the endosomes associated with SHORTROOT, and Kinesin G colocalise with the endoplasmic reticulum and that these endosomes move along the ER. By crossing the respective fluorescent markers into the background of mutants with perturbed organisation of the ER, they show that the intercellular transport of SHORTROOT is impaired. These data are consistent with a model, where a morphogenetic signal is transported between neighbouring cell layers along the ER channel crossing plasmodesmata in a microtubule-dependent fashion, which recalls the spread of the tobacco mosaic virus by virtue of its microtubulebinding movement protein (Ouko et al. 2010). In both cases, there is a cross-connection with actin-Kinesin G, through its $\mathrm{CH}$ domain should also be able to interact with actin, and actin plays also a central role for the organisation of the ER.

When two evolutionarily distant systems use the same functional principle - a minus-end-directed microtubule motor which cross-links to actin filaments - assembled from completely different molecular components, there must be a common selective principle. Both systems are related to signalling; in both systems, this signalling displays a distinct directionality: The centrosome recruits components by centripetal dynein-driven transport. In plant cells, where neither centrosomes nor dyneins are available and where the nuclear envelope acts as major microtubule-organising centre, minus-end-directed transport by $\mathrm{KCH}$ kinesins can be used to recruit a transcription factor to its site of action, in the nucleus of the endodermal precursor cell. The common principle behind this case of convergent evolution is the fact that minusend-directed motors allow reading out the history of a microtubule and, thus, organising cellular space dependent on activity (i.e. on time).

\section{References}

Allan V (1994) Dynactin: portrait of a dynein regulator. Curr Biol 4: 1000-1002

Clark J, Giniger E, Ruohola-Baker H, Jan LY, Jan YN (1994) Transient posterior localization of a kinesin fusion protein reflects anteroposterior polarity of the Drosophila oocyte. Curr Biol 4: 289-293

Fokin A, Zhapparova O, Burakov A, Nadezhdina ESS (2019) Centrosome-derived microtubule radial array, PCM-1 protein, and primary cilia formation. Protoplasma current issue

Hyman AA, Mitchison TJ (1991) Two different microtubule based motor activities with opposite polarities in kinetochores. Nature 351:206211

Nakajima K, Sena G, Nawy T, Benfey PN (2001) Intercellular movement of the putative transcription factor SHR in root patterning. Nature 413:307-311

Ouko MO, Sambade A, Brandner K, Ahad A, Heinlein M, Nick P (2010) Tobacco mutants with reduced microtubule dynamics are less susceptible to TMV. Plant J 62:829-839

Spiegelman Z, Lee CM, Gallagher KL (2018) KinG is a plant-specific kinesin that regulates both intra-and intercellular movement of SHORT-ROOT. Plant Physiol 176:392-405

Spiegelman Z, Wu SH, Gallagher KL (2019) A role for the endoplasmic reticulum in the cell-to-cell movement of SHORT-ROOT. Protoplasma, current issue

Supp DM, Witte DP, Potter SS, Brueckner M (1997) Mutation of an axonemal dynein affects left-right asymmetry in inversus viscerum mice. Nature 389:963-966

Publisher's note Springer Nature remains neutral with regard to jurisdictional claims in published maps and institutional affiliations. 